\title{
Laboratory Blood Coagulation in Sudanese with Falciparum Malaria: A Glance of Change Outcomes
}

\author{
Bashir Abdrhman Bashir ${ }^{*}$ and Mohamed Seed Ahmed \\ Department of Hematology, Faculty of Medical Laboratory Sciences, Port Sudan Ahlia College, Port \\ Sudan, Sudan
}

*Corresponding author: Bashir Abdrhman Bashir, Department of Hematology, Faculty of Medical Laboratory Sciences, Port Sudan Ahlia College, Port Sudan, Sudan

\begin{abstract}
Background: Malaria is a potentially life-threatening disease caused by infection of erythrocytes with one of five different types of protozoan parasites of genus plasmodia. Falciparum species is an aggressive type associated with multiple alterations in hemostasis.

Patients and Methods: A cross-sectional descriptive study was undertaken to screen the effect of malaria infection on coagulation test results in adults with the falciparum malaria parasite. Forty-eight consecutive adults with falciparum malaria were studied along with controls. All were subjected for the screening of prothrombin time (PT), partial thromboplastin time (PTT), thrombin time (TT), and D-Dimer.

Results: Changes in the coagulation parameters in the form of augmented PT, PTT and/or TT were seen in $22.9 \%, 4.2 \%$ and $14.6 \%$ of patients, respectively, without bleeding manifestation. D-Dimer was high in $37.5 \%$ cases. Receiver operation curve and area under the curve (AUC) analyses revealed a reasonable consistency with the density of falciparum malaria using PT (AUC $=0.69$, sensitivity $=$ $78.0 \%$ and specificity $=69.0 \%$, cut-off $=14.3$ seconds), PTT (AUC $=0.50$, sensitivity $=58.3 \%$ and specificity $=62.0 \%$, cut-off $=28.5$ seconds), TT (AUC $=0.29$, sensitivity $=36.1 \%$ and specificity $=$ $55.0 \%$, cut-off $=15.7$ seconds $)$, and D-Dimer $(A U C=0.70$, sensitivity $=80.0 \%$ and specificity $=66.0 \%$, cut-off $=0.105 \mathrm{mg} / \mathrm{l})$.

Conclusion: Falciparum malaria has a profound hemostatic impact among malaria patients. PT and D-Dimer could be applied to predict the falciparum malaria in clinical course.
\end{abstract}

\section{Keywords}

Coagulation test, Falciparum malaria, PT, PTT, D-Dimer, Sudan

\section{Introduction}

Approximately, 212 million cases of malaria with 429,000 deaths were measured worldwide in 2015 according to the latest WHO estimation [1]. The total number of absolute cases with malaria in Sudan was 497946 cases based on the WHO 2011 estimations [2]. Malaria is switched to humans (host) by the snap of an infected female Anopheles mosquito. Most human infections occur by $P$. falciparum and $P$. vivax, and limited degree to $P$. ovale, $P$. malariae, and $P$. knowlesi additionally infect human [3]. The causative agent of the acute form of malaria is $P$. falciparum which is responsible for the highest morbimortality rates [4]. Most of the mortality rates were in Africa (90\%), followed by Southern East Asia (7\%), and (3\%) in the eastern Mediterranean area [5]. Malaria in Sudan is considered a major public health problem and accounted for $37 \%$ of all maternal mortality [6]. Malaria parasite (Plasmodium) overruns red blood cells as part of its asexual turn in the host (human). The Plasmodium matures in the erythrocytes to trophozoites and schizonts, then they disappear from the blood and stationed specifically in the vascular beds, this mechanism is called sequestration [7]. Abnormal bleeding is regarded as one of the major manifestations of $P$. falciparum malaria [8]. Plasmodium pathogenesis has been associated with both endothelial activation and injury in uncomplicated or fatal cases of malaria. However, the molecule receptors like E-selectin, P-selectin, intracellular adhesion molecules-1 (ICAM-1), and tissue factor (TF) have been stated in a previous study [9]. Importantly, malarial infection has been classified into mild, moderate, and severe according to the rate of parasitemia [10]. In regard to laboratory hemostasis, malaria infection changes are not in fact the same in all studies [11], which might be due to severity of infection and/or selection of the patients. Coagulation disorders and thrombocytopenia are commonly seen in

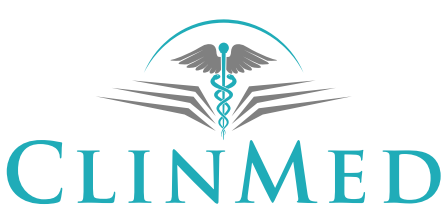

INTERNATIONAL LIBRARY

Citation: Bashir BA, Ahmed MS (2020) Laboratory Blood Coagulation in Sudanese with Falciparum Malaria: A Glance of Change Outcomes. Int J Blood Res Disord 7:056. doi.org/10.23937/24695696/1410056

Accepted: September 01, 2020: Published: September 03, 2020

Copyright: (C) 2020 Bashir BA, et al. This is an open-access article distributed under the terms of the Creative Commons Attribution License, which permits unrestricted use, distribution, and reproduction in any medium, provided the original author and source are credited. 
acute malarial infection, with $5 \%$ to $10 \%$ associated with bleeding. Several pathogenesis processes proposed may contribute to bleeding episodes in severe malaria, such as thrombocytopenia, impaired synthesis of blood clotting factors, and consumptive coagulopathy [8]. The classical disseminated intravascular coagulation (DIC) is encountered as a remarkable situation that appears in severe malaria [12]. Activation of the coagulation framework during malarial infection was stimulated by various procoagulants. These procoagulants are lysis of activated platelets, releasing of tissue factor under the action of endothelial cells injury or activated as well as the macrophage migration inhibitory factor (MIF), and interleukin-6 (IL-6) [13]. Due to the intrinsic pathway activation of coagulation, the complement system becomes indirectly activated and released bradykin in which could contribute to severe pathogenesis of malaria [14]. The elevation of D-Dimer was demonstrated in severe infection. The existence of fibrinogen/fibrin degradation products (FDPs) is mostly a compensatory mechanism indirectly to increase the formation of fibrin through malaria infection [15]. This study was designated to examine the laboratory coagulation state in falciparum malaria and the extent of abnormalities.

\section{Patients and Methods}

The present a cross-sectional study was conducted from October to November 2017 at Hematology department laboratories, Port Sudan Ahlia College, Sudan. 48 febrile patients with compatible features of falciparum malaria along with 48 well healthy individuals as control were enrolled. The following blood coagulation tests were estimated; PT, PTT, TT, and D-Dimer.

\section{Inclusion and exclusion}

The study populations included febrile confirmed positive falciparum malaria diagnosed by blood film smear and rapid diagnosis test (RDT) underwent this study. All patients with abnormal coagulation and underlying disorders which probably cause similar complications such as dengue fever, enteric fever, or sepsis were excluded. Patients who received blood or blood product also excluded.

\section{Study samples}

Antecubital veins were chosen for blood sample collection. Thin and thick blood films were performed immediately followed by RDTs for checking malaria, then $2.7 \mathrm{ml}$ venous blood added to $3.2 \%$ tri-sodium citrate vacutainer tubes (Greetmed, Batch GT/13/340, China). Platelet poor plasma was obtained by centrifugation. Viral screening tests were done for the icteric sample to rule out viral hepatitis.

\section{Laboratory methods}

In vitro diagnosis PT and PTT were determined with a commercially available kit (Biomed diagnostics, Ger- many) on a semi-automated URIT Coagulation analyzer. TT was also measured with commercially kit using (Techonoclone, Vienna, Asturia) reagents. D-Dimer test was estimated quantitatively by NycoCard ${ }^{\circ}$ reagent and method using NycoCard ${ }^{\circ}$ READER II (SN 67498, Axis-Shield PoC AS, Oslo, Norway).

\section{Examination of the blood films}

Patients were exposed to point by point history and physical assessment. The conclusive determination of malaria was made on the observation of parasites in the blood. Quantitative buffy coat evaluation was performed to determine malaria density.

\section{Data analysis}

Statistical package for the social sciences (SPSS, version 24, IBN, USA) was selected for the appropriate analysis of data. Data were shown as means and standard deviations (SD) and the category variables were seen as percentages. Data were checked for normality using the Kolmogorov-Smirnov test. Non-parametric tests, Mann-Whitney $U$ were utilized to assess the differences between the cases of falciparum malaria and controls. The Chi-square and Fisher's exact tests were performed to highlight the significant of density associated with the outcome and a 95\% confidence interval was also calculated. Receiver operating characteristics (ROC) and area under the curve (AUC) was applied to determine the diagnostic cutoff values of the coagulation parameters based on test sensitivity and specificity. P-values of less than 0.05 were taken statistically significant.

\section{Ethical approval}

This study was considered by the Medical Laboratory College Review Board, Port Sudan Ahlia College. Written informed consent was taken from each member of the study.

\section{Results}

Forty-eight febrile patients confirmed for Plasmodium falciparum infection were enrolled in the study, 21 (43.8\%) were males and 27 (56.2\%) were females. The ages of all participants ranged between $17-65$ years (mean age: $42.58 \pm 11.1$ years). 48 healthy appearing individuals were also subjected, 23 (47.9\%) were males and 25 (52.1\%) were females aged between $18-76$ years (mean age: $33.18 \pm 14.6$ years). Basic characteristic findings of falciparum malaria cases and controls are mentioned in Table 1. PT, PTT, and TT values were prolonged in 11 (22.9\%), 2 (4.2\%), and 7 (14.6\%) of cases, respectively. No clinical evidence of bleeding episodes was observed in our patients. D-dimer values were increased in 18 (37.5\%) of patients with falciparum malaria. The highlighted outcomes of coagulation parameters among the density of falciparum malaria expressed significant with D-Dimer ( $\mathrm{P}<0.001)(95 \% \mathrm{Cl}$; 0.159-0.426) (Table 2). D-Dimer values were correlated positively 
Table 1: Basic characteristic variables of controls and cases of falciparum malaria.

\begin{tabular}{|l|l|l|l|}
\hline Variables & $\begin{array}{l}\text { Malaria cases } \\
\text { (n= 48) }\end{array}$ & $\begin{array}{l}\text { Control } \\
\text { (n= 48) }\end{array}$ & P \\
\hline Age (mean \pm SD) & $42.58 \pm 11.1$ & $33.18 \pm 14.6$ & $<0.001$ \\
\hline Sex (male) & $21(43.8 \%)$ & $23(47.9 \%)$ & $<0.001$ \\
(female) & $27(56.2 \%)$ & $25(52.1 \%)$ & 0.002 \\
\hline PTs & $15.0 \pm 2.4$ & $13.7 \pm 1.6$ & 0.994 \\
\hline PTTs & $29.4 \pm 8.44$ & $28.66 \pm 4.35$ & $<0.001$ \\
\hline TTs & $14.62 \pm 3.06$ & $16.46 \pm 2.37$ & $<0.001$ \\
\hline D-Dimer $\mathrm{mg} / \mathrm{l}$ & $0.43 \pm 0.44$ & $0.13 \pm 0.07$ & \\
\hline
\end{tabular}

Table 2: Coagulation changes outcome with density of falciparum malaria.

\begin{tabular}{|c|c|c|c|c|c|}
\hline Parameters & $\begin{array}{l}\text { Density }(+) \\
(n=36)\end{array}$ & $\begin{array}{l}\text { Density (++) } \\
(n=12)\end{array}$ & Fishers exact test & $\mathbf{p}$ & $95 \% \mathrm{Cl}$ \\
\hline $\begin{array}{l}\text { PT, n (\%) } \\
\text { Normal } \\
\text { Short } \\
\text { Prolong }\end{array}$ & $\begin{array}{l}27(56.3) \\
3(6.3 \%) \\
6(12.5 \%)\end{array}$ & $\begin{array}{l}6(12.5 \%) \\
1(2.1 \%) \\
5(10.4 \%)\end{array}$ & 0.152 & 0.195 & $0.541-2.192$ \\
\hline $\begin{array}{l}\text { PTT, n (\%) } \\
\text { Normal } \\
\text { Short } \\
\text { Prolong }\end{array}$ & $\begin{array}{l}17(35.4 \%) \\
18(37.8 \%) \\
1(2.1 \%)\end{array}$ & $\begin{array}{l}10(56.3 \%) \\
1(2.1 \%) \\
1(2.1 \%)\end{array}$ & 0.018 & 0.035 & - 1.964-3.481 \\
\hline $\begin{array}{l}\text { TT, n (\%) } \\
\text { Normal } \\
\text { Short } \\
\text { Prolong }\end{array}$ & $\begin{array}{l}32(66.7 \%) \\
1(2.1 \%) \\
3(6.3 \%)\end{array}$ & $\begin{array}{l}8(16.7 \%) \\
0 \\
4(8.4 \%)\end{array}$ & 0.127 & 0.094 & $-2.950--0.729$ \\
\hline $\begin{array}{l}\text { D-Dimer, n (\%) } \\
\text { Normal } \\
\text { High }\end{array}$ & $\begin{array}{l}30(62.5 \%) \\
6(12.5 \%)\end{array}$ & $\begin{array}{l}0 \\
12(25.0 \%)\end{array}$ & 0.000 & 0.000 & $0.159-0.426$ \\
\hline
\end{tabular}

Table 3: ROC curve analysis of coagulation tests in predicting the clinical course of malaria.

\begin{tabular}{|l|l|l|l|l|l|l|l|}
\hline Variables & AUC & P & Sensitivity (\%) & Specificity (\%) & Cut off & 95\% Cl \\
\hline PT & 0.690 & 0.002 & $78 \%$ & $69 \%$ & 14.3 & $0.579-0.794$ \\
\hline PTT & 0.500 & 0.994 & $58.1 \%$ & $62 \%$ & 28.5 & $0.381-0.618$ \\
\hline TT & 0.290 & 0.000 & $36.1 \%$ & $55 \%$ & 15.7 & $0.177-0.387$ \\
\hline D-Dimer & 0.700 & 0.001 & $80 \%$ & $66 \%$ & 0.105 & $0.586-0.798$ \\
\hline
\end{tabular}

with PT values ( $r: 0.264 / \mathrm{P}<0.009)$. So, D-dimer and PTT values were correlated positively with the density of falciparum malaria parasitemia $(r: 0.642 / P<0.000$ and $r: 0.326 / P<0.024$, respectively).

ROC curve analysis and calculation of AUC for PT, PTT, TT, and D-Dimer were conducted for prediction influenced of coagulation tests owing to the density of falciparum malaria parasites. The outcome appeared in Table 2, in which there is reasonable consistency with the density of falciparum malaria using PT (AUC $=0.69$, sensitivity $=78.0 \%$ and specificity $=69.0 \%$, cut-off $=14.3$ second), PTT (AUC $=0.50$, sensitivity $=58.3 \%$ and specificity $=62.0 \%$, cut-off $=28.5$ second $)$, TT (AUC $=0.29$, sensitivity $=36.1 \%$ and specificity $=55.0 \%$, cut-off $=15.7$ second), and D-Dimer ( $A \cup C=0.70$, sensitivity $=80.0 \%$ and specificity $=66.0 \%$, cut-off $=0.105 \mathrm{mg} / \mathrm{l})$, (Table 3 ).

\section{Discussion}

In falciparum malaria, there is an expanded turnover of the coagulation course that may end up with consumptive coagulopathy, which may prompt the advancement of DIC. The actual action isn't yet known. However, it has been guessed that tainted erythrocytes have genius coagulant activity on account of changes in the lipid dispersion over the inner and outer surfaces of the infected erythrocyte velum [16]. Endothelial injury may likewise be significant in advancing initiation of the coagulation course. Even though the disruption in coagulation parameters happens generally in cases with extreme malaria, and hemorrhage happens in very scanty cases [17].

In this study, the male to female ratio was 0.8:1.2. This ratio highlighted that females are more likely af- 
fected than males, consequently to the residence pattern i.e. Females are uncovered to mosquito bites indoors while males are less exposed. These findings are differently violated with Jayashankar, et al. probably due to the population differences [18]. PT was excessively increased in $22.9 \%$ of the total falciparum malaria cases. In previous studies performed by Datta, et al. [19] and Jayashankar, et al. prothrombin time was increased by $11.6 \%$ and $37 \%$, respectively. Chukwuanukwu, et al. was also reported that PT was prolonged in the cases of falciparum malaria, which resembled the indication of the present study [20].

In the current study, PTT was seen prolonged in $4.2 \%$ of falciparum malaria cases. In a study led by Kashinkunti, et al. PTT was increased in $31 \%$ of the falciparum malaria patients [21], this was less than what we saw in our study. On the other hand, when contrasted with controls, the falciparum malaria cases likewise had prolonged TT and raised D-Dimer levels, which were of statistical measurable significance. These findings were also found earlier [19]. In other words, apoptotic cells conceivably support the coagulation course and seem to take an interest in inflammation in vivo. However, it may hypothesize that in malaria there is an apoptotic-coagulation-inflammation unevenness. Thus, monocytes may assume a significant role in the sickness pathogenesis or potentially add to the procoagulant/inflammatory tonus saw in falciparum malaria, as portrayed for sepsis. Of course, malaria has been viewed as a provocative sepsis-like disorder [22]. Ultimately, it was suggested that the number of patients who perceive with a high density of parasitemia had a lot of frequency of alterations in coagulation tests more so as for PT and D-Dimer. Furthermore, could elucidate the clinical course of the disease as expanded by ROC curve.

The consequences of our outcomes ought to be analyzed circumspectly as a result of the distinction in the malarial sickness endemicity/immunity in various settings. This work has major restrictions that may sway our results, the modest population number, and neglecting of clinical remarks. Besides, the diagnostic tools depend upon the microscopic examination and RDT. Many factors that may impact the coagulation tests, we tried to preclude as much as possible. Nonetheless, further surveys are required to verify the diagnosis.

\section{Conclusion}

This study indicates that the occurrences of coagulopathy were higher in cases with falciparum malaria without bleeding highlight, where the coagulation tests were adjusted when contrasted with controls. Additionally, the number of cases with PT and D-Dimer coagulopathy was significantly raised in cases with a high density of parasitemia.

\section{References}

1. World health organization (2016) World Malaria Report 2016.

2. Hussein HH, Eissan FH, Awadalla KE (2017) Statistical methods of predicting malaria incidence using data from sudan. Malar Res Treat 2017: 1-9.

3. Singh B, Daneshvar C (2013) Human infections and detection of Plasmodium knowlesi. Clin Microbiol Rev 26: 165-184.

4. Leffler EM, Band G, Busby G, Kivinen K, Le Q S, et al. (2017) Resistance to malaria through structural variation of red blood cell invasion receptors. Science 356: eaam6393.

5. World Health Organization (2013) World malaria report 2013. Geneva: WHO Press.

6. Ali A, Khojali A, Okud A, Adam G, Adam I (2011) Maternal near-miss in a rural hospital in Sudan. BMC Pregnancy Childbirth 11: 48.

7. Samuel C Wassmer, Terrie E Taylor, Pradipsinh K Rathod, Saroj K Mishra, Sanjib Mohanty, et al. (2015) Investigating the Pathogenesis of Severe Malaria: A Multidisciplinary and Cross-Geographical Approach. Am J Trop Med Hyg 93: 4256.

8. Angchaisuksiri $\mathrm{P}$ (2014) Coagulopathy in malaria. Thromb Res 133: 5-9.

9. Alho RM, Machado VK, Val FF, Fraiji NA, Alexandre MA, et al. (2017) Alternative transmission routes in the malaria elimination era: an overview of transfusion-transmitted malaria in the Americas. Malar J 16: 78.

10. Bartoloni A, Zammarchi $L$ (2012) Clinical aspects of uncomplicated and severe malaria. Mediterr J Hematol Infect Dis 4: e2012026.

11. Antinori S, Corona A, Ridolfo AL, Galimberti L, Davide Ricaboni, et al. (2016) Imported Plasmodium vivax malaria with severe thrombocytopaenia: can it be severe malaria or not? Malar J 15: 105

12. Sailo L, Pradhan D, Nongthombam R, Bhattacharyya $P$ (2014) Disseminated intravascular coagulation in malaria: A case report. Niger Med J 55: 171-172.

13. Bridges DJ, Bunn J, van Mourik JA, Grau G, Preston RJS, et al. (2010) Rapid activation of endothelial cells enables Plasmodium falciparum adhesion to platelet decorated von Willebrand factor strings. Blood 115: 1472-1474.

14. O'Sullivan JM, Preston RJ, O'Regan N, O'Donnell JS (2016) Emerging roles for hemostatic dysfunction in malaria pathogenesis. Blood 127: 2281-2288.

15. World Health Organization, Fact sheet (2019) World malaria report 2019.

16. Muhindo Mavoko H, Kalabuanga M, Delgado-Ratto C, Maketa V, Mukele R, et al. (2016) Uncomplicated clinical malaria features, the efficacy of Artesunate-Amodiaquine and their relation with multiplicity of infection in the Democratic Republic of Congo. PLoS One 11: e0157074.

17. Gillriea MR, May Ho (2017) Dynamic interactions of Plasmodium spp. with vascular endothelium. Tissue Barriers 5: e1268667.

18. Jayashankar CA, Pinnelli VB, Prabhu R (2016) Alteration of coagulation profile in malaria patients and its correlation with degree of parasitemia: a prospective study. Int J Adv Med 3: 388-392.

19. Datta S, Roul LD, Das S, Panda JK, Pattanayak NC, et al. (2011) A study of the Coagulation profile in falciparum malaria. Orissa Physicians Journal 7: 7-12. 
20. Chukwuanukwu RC, Ukaejiofo EO, Ele PU, Onyenekwe CC, Chukwuanukwu TO, et al. (2016) Evaluation of some haemostatic parameters in falciparum malaria and HIV co-infection. Br J Biomed Sci 73: 168-173.

21. Kashinkunti M, Alevoor S (2014) Clinical, Hematological and coagulation proflile in malaria. Sch J App Med Sci 2: 584-588.

22. Riedl J, Mordmüller B, Koder S, Ingrid Pabinger, Peter G Kremsner, et al. (2016) Alterations of blood coagulation in controlled human malaria infection. Malar J 15. 